\title{
Qualitative Mathematical Analysis of the Richards Equation
}

\author{
B. H. GILDING \\ Faculty of Applied Mathematics, University of Twente, P.O. Box 217, 7500 AE Enschede, The Netherlands
}

(Received: 16 October 1989; in final form: 5 October 1990)

\begin{abstract}
The Richards equation is widely used as a model for the flow of water in unsaturated soils. For modelling one-dimensional flow in a homogeneous soil, this equation can be cast in the form of a specific nonlinear partial differential equation with a time derivative and one spatial derivative. This paper is a survey of recent progress in the pure mathematical analysis of this last equation. The emphasis is on the interpretation of the results of the analysis. These are explained in terms of the qualitative behaviour of the flow of water in an unsaturated soil which is described by the Richards equation.
\end{abstract}

Key words. Mathematical analysis, partial differential equation, nonlinear, diffusion, advection, FokkerPlanck, qualitative behaviour, unsaturated soils, wetting-fronts.

\section{Nomenclature}

\section{Roman letters}

a coefficient in second-order diffusion term of equation

$b$ coefficient in first-order advection term of equation

$D$ soil-moisture diffusivity $\left[\mathrm{L}^{2} \mathrm{~T}^{-1}\right]$

$h$ pressure head [L]

$H$ quarter-plane domain for Cauchy-Dirichlet problem [L] $\times[\mathrm{T}]$

$K$ hydraulic conductivity scalar [ $\left.\mathrm{LT}^{-1}\right]$

K hydraulic conductivity tensor $\left[\mathrm{LT}^{-1}\right]$

$q$ soil-moisture flux scalar $\left[\mathrm{LT}^{-1}\right]$

q soil-moisture flux vector $\left[\mathrm{LT}^{-1}\right]$

$r$ dummy variable

$R$ rectangle $[\mathrm{L}] \times[\mathrm{T}]$

$s \quad$ dummy variable

$s^{*}$ representative value of dummy variable

$S$ half-plane domain for Cauchy problem [L] $\times[\mathrm{T}]$

$t$ time [T]

$u$ unknown solution of partial differential equation

$u_{0}$ initial-value function

$v \quad$ soil-moisture velocity scalar $\left[\mathrm{LT}^{-1}\right]$

v soil-moisture velocity vector $\left[\mathrm{LT}^{-1}\right]$ 
$x$ distance, position [L]

$z$ elevation [L]

Greek letters

$\alpha \quad$ angle to horizontal $[-]$

$\zeta$ interface [L]

$\theta$ water content [-]

$\theta_{r} \quad$ irreducible water content $[-]$

$\theta_{s} \quad$ water content at saturation [-]

$\lambda$ directional parameter $[-]$

$\sigma$ test parameter in criterion for finite speed of propagation [LT $\left.{ }^{-1}\right]$

$\sigma_{0}$ minimum speed of propagation $\left[\mathrm{LT}^{-1}\right]$

$\phi \quad$ test function

$\Omega \quad$ arbitrary domain $[\mathrm{L}] \times[\mathrm{T}]$

\section{Introduction}

Neglecting the effects of heat and density gradients, sorption, osmosis and plant abstraction, the flow of water in a soil may be described by Darcy's law

$$
\mathbf{q}=-\mathbf{K} \operatorname{grad}(h+z)
$$

and conservation of mass

$$
\frac{\partial \theta}{\partial t}+\operatorname{div} \mathbf{q}=0 .
$$

Here $\mathbf{q}$ denotes soil-moisture flux, $\mathbf{K}$ is the hydraulic conductivity tensor, $h$ pressure head, $z$ elevation, $\theta$ water content, $t$ time and the differential operators 'grad' and 'div' are defined in terms of a fixed coordinate system. The vector $\mathbf{q}$ denotes the bulk flux in the porous medium and is related to the velocity $\mathbf{v}$ of the moisture by

$$
\mathbf{q}=\theta \mathbf{v} .
$$

Supposing that the soil is isotropic and that the flow is essentially one-dimensional in a direction which is inclined at an angle $\alpha$ to the horizontal, (1) and (2) become

$$
q=-K \frac{\partial}{\partial x}(h+\lambda x)
$$

and

$$
\frac{\partial \theta}{\partial t}=-\frac{\partial}{\partial x} q,
$$

respectively. In (3) and (4), $q$ and $K$ are scalars, $x$ denotes distance in the direction of flow, and $\lambda=\sin \alpha$ (see Figure 1). Combining (3) with (4) yields

$$
\frac{\partial \theta}{\partial t}=\frac{\partial}{\partial x}\left(K \frac{\partial h}{\partial x}+\lambda K\right) .
$$




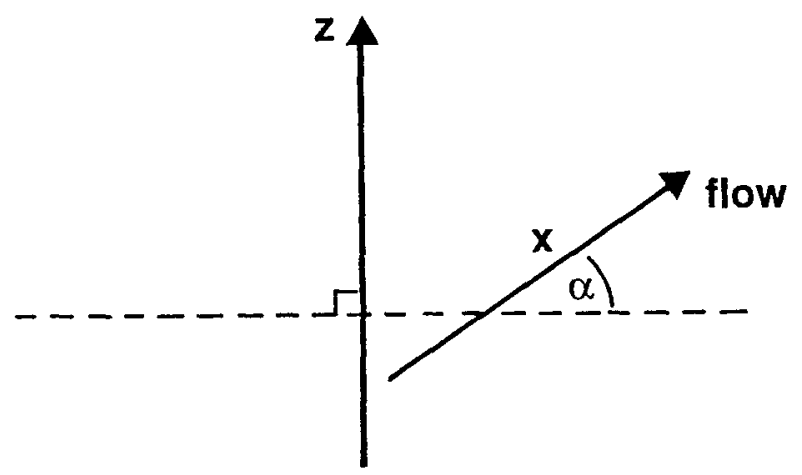

Fig. 1. Direction of flow.

If, furthermore, the soil is supposed to be homogeneous and unsaturated, and hysteresis may be neglected, then $K$ and $h$ may be considered as single-valued functions of $\theta$. In this case, Equation (5) may be rewritten as

$$
\frac{\partial \theta}{\partial t}=\frac{\partial}{\partial x}\left(D(\theta) \frac{\partial \theta}{\partial x}\right)+\lambda \frac{\partial}{\partial x}(K(\theta)),
$$

where

$$
D(\theta)=K(\theta) \frac{\mathrm{d} h}{\mathrm{~d} \theta}(\theta)
$$

denotes soil-moisture diffusivity $[3,5,9,20,24]$.

Equation (6) is known as the one-dimensional Richards equation formulated in terms of water content [21]. In certain circles [4, 20], this equation is also referred to as the nonlinear Fokker-Planck equation because of its resemblance to the celebrated (linear) Fokker-Planck equation occurring in the theory of statistical mechanics [8]. The first-order term on the right-hand side of (6) is frequently called the gravity term. If the flow is horizontal, then $\lambda=0$, whilst if the flow is vertical in an upwards direction $\lambda=1$, and if the flow is vertical in a downward direction $\lambda=-1$.

Typical illustrations of the $h(\theta)$ and $K(\theta)$ relationships as found in standard references $[3,9,20,24]$ are shown in Figures 2 and 3 . Here $\theta_{\mathrm{s}}$ denotes the water content at saturation and $\theta_{r}$ the irreducible water content. The irreducible water content is taken to be zero in the majority of references.

In unsaturated soils, the pressure head is negative and is equated in magnitude with soil-mositure tension or suction. As the water content in a soil progressively increases, it is generally conceived $[9,24]$ that the smaller pores in which the capillary forces are the greatest are filled before the larger pores. Hence, pressure head $h$ is an increasing function of water content $\theta$. Contemporaneously, since the larger pores in the soil are the most effective conductors, the hydraulic conductivity increases as the water content increases and does so at a progressively greater rate. 


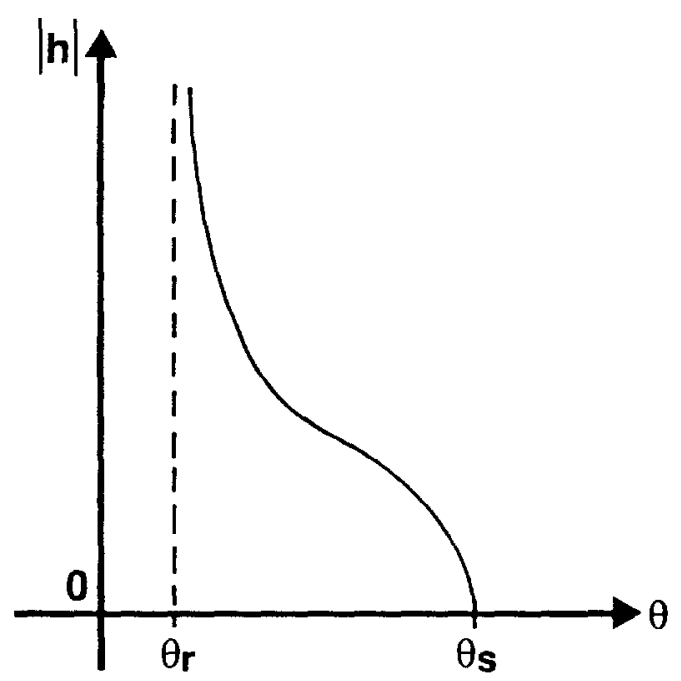

Fig. 2. Typical $h(\theta)$ relationship.

Consequently, hydraulic conductivity $K$ is pictured as an increasing convex function of $\theta$. This function assumes the value zero when the water content assumes its irreducible value.

A typical illustration $[9,24]$ of the soil-mositure diffusivity relationship including moisture transport in the vapour phase is shown in Figure 4.

The purpose of this paper is to review recent progress [15-17] in the pure mathematical analysis of the equation

$$
u_{\mathrm{t}}=(a(u))_{\mathrm{xx}}+(b(u))_{\mathrm{x}}
$$

in which subscripts denote partial differentiation, and to relate this to the study of Equation (6).

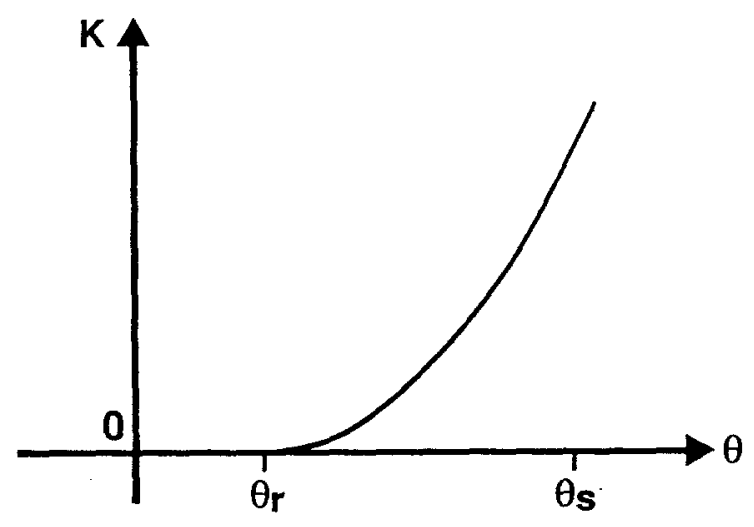

Fig. 3. Typical $K(\theta)$ relationship. 


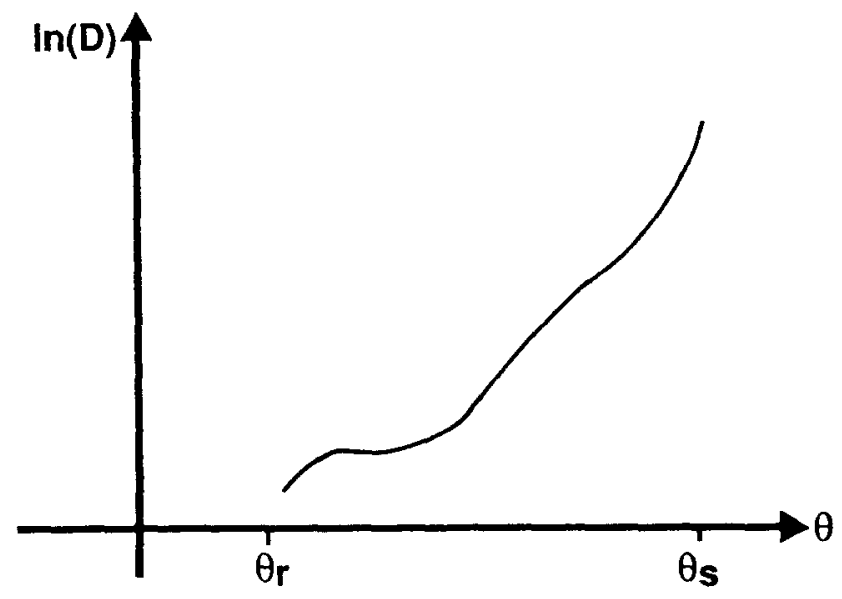

Fig. 4. Typical $D(\theta)$ relationship.

The mathematical analysis of (7) has been predominantly concerned with nonnegative solutions of the equation under the assumptions that the coefficients $a(s)$ and $b(s)$ are defined and continuous for nonnegative arguments $s, a(s)$ and $b(s)$ are twice continuously differentiable for positive arguments $s$ with second derivatives which are what is mathematically known as locally Hölder continuous,

$$
a^{\prime}(s)>0 \text { for } s>0
$$

and

$$
a(0)=b(0)=0 .
$$

Identifying the unknown $u$ in (7) with $\theta-\theta_{\mathrm{r}}$ in (6), $a$ with the indefinite integral of $D$, and $b$ with $\lambda K$, Equation (7) can be seen to be equivalent to Equation (6). The hypothesis (8) is equivalent to the positivity of the soil-moisture diffusivity coefficient for water contents above the irreducible level. The hypothesis $a(0)=0$ in (9) is of no significance since this amounts to no more than a fixing of the constant of integration in the identification of $a$ with the integral of $D$. The hypothesis $b(0)=0$ though is equivalent to the datum that the unsaturated hydraulic conductivity tends to zero as the water content approaches its irreducible value.

Of particular note in what follows is that the quantity $-\left((a(u))_{\mathrm{x}}+b(u)\right)$ can be identified with the flux $q$ in the derivation of the Richards equation and, subsequently, that $-\left((a(u))_{\mathrm{x}}+b(u)\right) / u$ can be identified with the one-dimensional soilmoisture velocity $v$ for the flow described by this equation.

The survey of the recent mathematical work on Equation (7) will be addressed to the topic of what this says about the acceptability of (6) as a model of the flow of water in unsaturated soils. 


\section{Solvability}

For the soil-physicist or other practitioner considering using the Richards equation (6) for modelling the flow of water in an unsaturated soil, the first question which must spring to mind is: Given suitable initial and boundary conditions, is there a solution to the equation? For the mathematician this question is the question of existence.

The blunt answer to this question is: No! For many years now it has been known that even with the most regular initial and boundary data it is not possible to find a classical solution to an equation of the type (7) without particularly restrictive conditions on the coefficients $a$ and $b$ which are unrealistic in the present situation [19]. By a classical solution, one means a function $u(x, t)$ for which one can define the partial derivatives $\partial u / \partial t, \partial^{2}(a(u)) / \partial x^{2}$ and $\partial(b(u)) / \partial x$ at every point in the domain under consideration in the sense of the limit process with which partial derivatives are defined in any introductory calculus course, and for which one can equate them by (7) at every point in the domain. However, the answer to the question is: Yes; if one considers solutions defined in a suitable abstract mathematical sense.

There are many ways in which the notion of a solution of (7) can be mathematically abstracted $[1,6,7,10,14,16,22]$. One gambit is to observe that if a function $u(x, t)$ is a classical solution of Equation (7) in some domain $\Omega$, then taking any rectangle $R=\left(x_{1}, x_{2}\right) \times\left(t_{1}, t_{2}\right]$ in $\Omega$ and any nonnegative function $\phi$ which is defined and sufficiently smooth in $R$ and which vanishes for $x=x_{1}$ and $x=x_{2}$, multiplying (7) by $\phi$ and integrating by parts one obtains the identity

$$
\begin{aligned}
\iint_{R}\left\{u \phi_{t}+a(u) \phi_{\mathrm{xx}}-b(u) \phi_{\mathrm{x}}\right\} \mathrm{d} x \mathrm{~d} t \\
=\int_{x_{1}}^{x_{2}}\left\{u\left(x, t_{2}\right) \phi\left(x, t_{2}\right)-u\left(x, t_{1}\right) \phi\left(x, t_{1}\right)\right\} \mathrm{d} x+ \\
\quad+\int_{t_{1}}^{t_{2}}\left\{a\left(u\left(x_{2}, t\right)\right) \phi_{\mathrm{x}}\left(x_{2}, t\right)-a\left(u\left(x_{1}, t\right)\right) \phi_{\mathrm{x}}\left(x_{1}, t\right)\right\} \mathrm{d} t .
\end{aligned}
$$

A generalized solution of Equation (7) in a domain $\Omega$ is defined as a function $u(x, t)$ which is continuous, nonnegative, and bounded in $\Omega$ and on the boundary of $\Omega$ and which satisfies the integral identity (10) for any rectangle $R$ and function $\phi$ with the afore-stated properties. In this way, any classical solution is a generalized solution. However, the converse is not true.

With the above notion of a generalized solution, various existence theorems for initial and boundary value problems for Equation (7) have been proven [16]. Thus if one considers solutions in this sense, the question of existence has been answered.

The next most obvious question is: Given suitable initial and boundary conditions, is there only one solution? For the mathematician this is the question of uniqueness.

The answer to this question is also in the affirmative considering the generalized solutions of Equation (7). 
For the mathematician the situation is now clear-cut. For fairly respectable problems there is one and only one solution. The natural question that the soil-physicist will then ask though is: Does the generalized solution have any physical relevance? To answer this question one may consider the mathematical aspect of regularity.

To discuss the regularity of generalized solutions of Equation (7), for simplicity, attention will be restricted to the Cauchy problem; this is to say, the problem of solving (7) in the domain

$$
S=\{(x, t):-\infty<x<\infty, 0<t<\infty\}
$$

subject to the initial condition

$$
u(x, 0)=u_{0}(x) \text { for }-\infty<x<\infty,
$$

where $u_{0}$ is a given function. It is hypothesized that $u_{0}$ is nonnegative, continuous and bounded.

Inherent in the definition of a generalized solution $u(x, t)$ of this problem is the property:

$-u$ is bounded and continuous in the domain $\bar{S}=\{(x, t):-\infty<x<\infty$, $0 \leqslant t<\infty\}$.

The following has also been proven $[15,16]$ :

- $(a(u))_{\mathrm{x}}+b(u)$ is bounded in the mathematical sense of distributions in the set $\left\{(x, t):-\infty<x<\infty, t_{0} \leqslant t<\infty\right\}$ for every $t_{0}>0$.

$-(a(u))_{\mathrm{x}}+b(u)$ is bounded in the sense of distributions in $S$ if this quantity is initially bounded for $t=0$ in the sense of distributions.

- $(a(u))_{\mathrm{x}}+b(u)$ is continuous in $S$ and $\left((a(u))_{\mathrm{x}}+b(u)\right) / u$ is bounded in the sense of distributions in $S$ if the latter quantity is initially bounded in the sense of distributions.

$-(a(u))_{\mathrm{x}}+b(u)$ is continuous in the set $\left\{(x, t):-\infty<x<\infty, t_{0} \leqslant t<\infty\right\}$ and $\left((a(u))_{\mathrm{x}}+b(u)\right) / u$ is bounded in the sense of distributions in this set for every $t_{0}>0$ if $a^{\prime}(s) / s$ is integrable and $b(s) / s$ is bounded in a neighbourhood of $s=0$. (Strictly speaking this is a simplification of the results. The reader is referred to [15] for details.)

$-u$ is a classical solution of Equation (7) at any point $x$ and time $t$ in $S$ where $u(x, t)>0$.

An explanation of what is meant by 'in the sense of distributions' may be found for example in [23].

To interpret the above results for the Richards equation, recall that the quantity $-\left((a(u))_{\mathrm{x}}+b(u)\right)$ is identified with the flux $q$ in the derivation of (6), and that the quantity $-\left((a(u))_{x}+b(u)\right) / u$ is analogous to the soil-moisture velocity $v$. In this light, the mathematical regularity results may be explained as indicating that for the 
appropriately-defined generalized solutions of (6):

- The water content $\theta$ is continuous and bounded.

- The soil-moisture flux $q$ becomes bounded after an infinitestimal time span irrespective of the initial soil-moisture distribution.

- The soil-moisture flux $q$ remains bounded if one begins with an initial soil-moisture distribution with bounded flux.

- The soil-moisture flux $q$ is continuous and the soil-moisture velocity $v$ remains bounded if one begins with an initial soil-moisture distribution with bounded velocity.

- Irrespective of the initial soil-moisture distribution, the soil-moisture flux $q$ becomes continuous and the soil-moisture velocity $v$ becomes bounded after an infinitesimal time-span if the soil-moisture diffusivity $D(\theta)$ and hydraulic conductivity $K(\theta)$ relationships satisfy certain constraints. (The significance of these constraints is a topic which will be returned to later.)

- The equation is solvable in the classical sense after all at any point and instant where the water content is not irreducible.

Thus, albeit that one has to consider solutions of (6) in some abstract mathematical sense, given appropriate initial conditions, these solutions turn out to possess all the properties which one would physically consider necessary, viz. continuous water content, continuous flux and bounded velocity.

Furthermore, since Equation (6) is trivially satisfied by the constant solution $\theta=\theta_{\mathrm{r}}$ inside sub-domains where the water content is irreducible, the only points in which the equation is not solvable in the classical sense and which, therefore, necessitate the introduction of the mathematical notion of a generalized solution, are those which verge on areas where the water content approaches its irreducible value.

With hindsight this is of course wholly consistent with the expounded derivation of (6), since the expressions $h(\theta)$ and $\mathrm{d}(h(\theta)) / \mathrm{d} \theta$ only have a meaning if the water content is above its irreducible level.

\section{The Phenomenon of Wetting-Fronts}

It should be evident from the previous section that the crucial difficulty with the mathematics of Equation (6) is occasioned by the possible occurrence of points demarcating regions in which the water content is above its irreducible level. Since in those areas where the water content is irreducible the medium is to all intents and purposes dry, such a point is equivalent to the physical phenomenon of a wettingfront. The question to be asked is then: Do wetting-fronts really occur? This question can be epitomized by supposing that one takes a dry soil and introduces water into a restricted area. One would then like to know whether at any later time the water still occupies a restricted area in an otherwise dry medium, or whether the distinction between wet and dry regions immediately disappears. (For an illustration in a situation where the irreducible water content $\theta_{\mathbf{r}}=0$, see Figure 5.) 


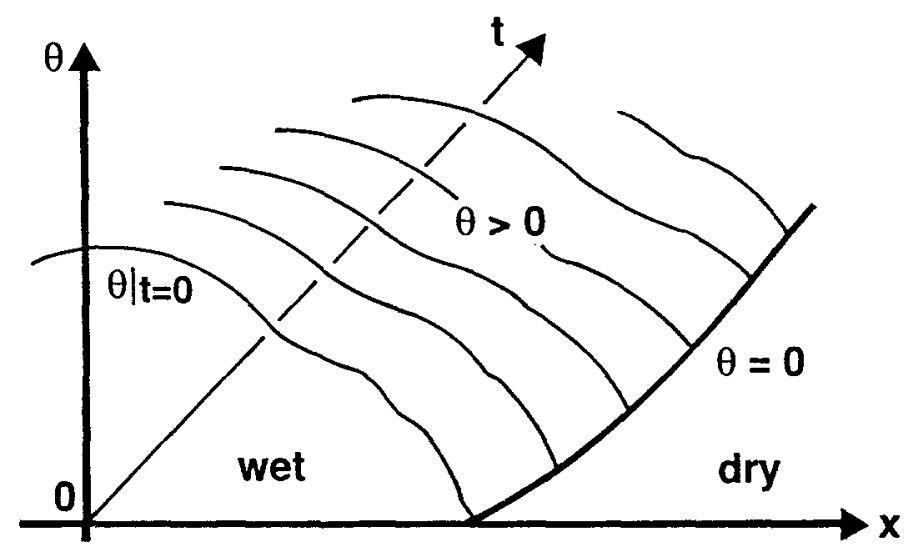

Fig. 5. Visualization of a wetting-front.

The latter question can be translated as follows. Consider the solution $u(x, t)$ of the Cauchy problem for Equation (7) and define

$$
\zeta(t)=\sup \{x:-\infty<x<\infty, u(x, t)>0\}
$$

for all $t \geqslant 0$. This is to say, let $\zeta(t)$ denote the least upper bound of the points where the solution is positive at any time $t$. Then if

$$
-\infty<\zeta(0)<\infty,
$$

is it true that

$$
-\infty<\zeta(t)<\infty \text { for any } t>0 \text { ? }
$$

If this is the case one speaks of finite speed of propagation in mathematical terms and refers to the point $\zeta(t)$ which corresponds to a wetting-front as an interface. The expression 'finite speed of propagation' may be declared in terms of the Richards' equation by observing that if there are no wetting-fronts in the sketched situation, then water must have instantaneously penetrated arbitrarily far into the medium and, thus, have progagated with an infinite speed.

It has been proven $[15,18]$ that in the above-mentioned context Equation (7) displays finite speed of propagation if and only if

$$
a^{\prime}(s) / \max \{s, b(s)\} \text { is integrable from } 0 \text { to } s^{*}
$$

and there is a constant $\sigma$ such that

$$
-b(s)<\sigma s \text { for } 0<s \leqslant s^{*}
$$

for some $s^{*}>0$.

This result has been obtained with the aid of two mathematical tools which have been established for generalized solutions of (7). The first of these is the comparison principle. This says that given two generalized solutions of Equation (7), $u_{1}$ and $u_{2}$ 
say, in some domain $\Omega$ of the form $\left(x_{1}, x_{2}\right) \times\left(t_{1}, t_{2}\right]$, if $u_{1} \geqslant u_{2}$ at every point on the boundary of $\Omega$ excluding the line $t=t_{2}$ then $u_{1} \geqslant u_{2}$ in the whole of $\Omega$. The other tool is the integral identity

$$
\int_{-\infty}^{\infty} u(x, t) \mathrm{d} x=\int_{-\infty}^{\infty} u_{0}(x) \mathrm{d} x \text { for any } t>0
$$

for generalized solutions $u(x, t)$ of the Cauchy problem for Equation (7). Both of these tools may be couched in physical terms.

The comparison principle basically says that given two identical soil samples, if the first is initially wetter than the other and is continually maintained wetter on the perimeter than the other, then at any later time this sample will also be wetter at every point in the interior. The argument may be refined by the observation that if one also injects water into the first sample or abstracts it from the second, the conclusion remains valid. In mathematical terms this is tantamount to the comparison principle for generalized supersolutions and subsolutions.

Noting the analogy between $u$ and $\theta$ and recollecting that in the derivation of Equation (6) it has implicitly been assumed that the water density is constant, the integral identity (15) is no more than a statement of conservation of mass for the soil water.

Returning to the question of the existence of wetting-fronts, recall that $u$ in (7) is equivalent to $\theta-\theta_{\mathrm{r}}$ in (6), the derivative $a^{\prime}$ is analogous to the diffusivity coefficient $D(\theta)$, and $b$ is equivalent to $\lambda K(\theta)$. Whilst, $K(\theta)>0$ for $\theta>\theta_{\mathrm{r}}$, and $\lambda=1$ if the flow is directed upwards, $\lambda=0$ if the flow is horizontal, and $\lambda=-1$ if the flow is directed downwards. In this light, the somewhat esoteric condition (13), (14) infers that in one-dimensional flow wetting-fronts can occur in an upward direction if and only if

$$
D(\theta) / \max \left\{\theta-\theta_{\mathbf{r}}, K(\theta)\right\} \text { is integrable from } \theta_{\mathbf{r}} \text { to } \theta_{\mathbf{s}} .
$$

Whilst wetting-fronts can occur in a horizontal direction if and only if

$$
D(\theta) /\left(\theta-\theta_{\mathrm{r}}\right) \text { is integrable from } \theta_{\mathrm{r}} \text { to } \theta_{\mathrm{s}} \text {. }
$$

Finally, wetting fronts can occur in a downward direction if and only if (17) holds and $K(\theta)$ lies under a straight-line when visualized as in Figure 6. Note that in this case, (16) and (17) are equivalent.

Consequently if one believes in wetting-fronts in all directions the soil-moisture diffusivity relationship must satisfy (17) and the $K(\theta)$ relationship must appear as in Figure 6. Observe though that if the $K(\theta)$ curve is convex, which experiment and argument have indicated is the case, the latter criterion is automatically satisfied.

It is interesting to note that the last-mentioned constraints on the relations $D(\theta)$ and $K(\theta)$ are precisely those under which the optimal regularity results discussed earlier were obtained. Thus, under these constraints, not only can one conclude that wetting-fronts are admissible but also one can conclude that, in any given soil profile, the soil-moisture flux will be continuous and the soil-moisture velocity will 


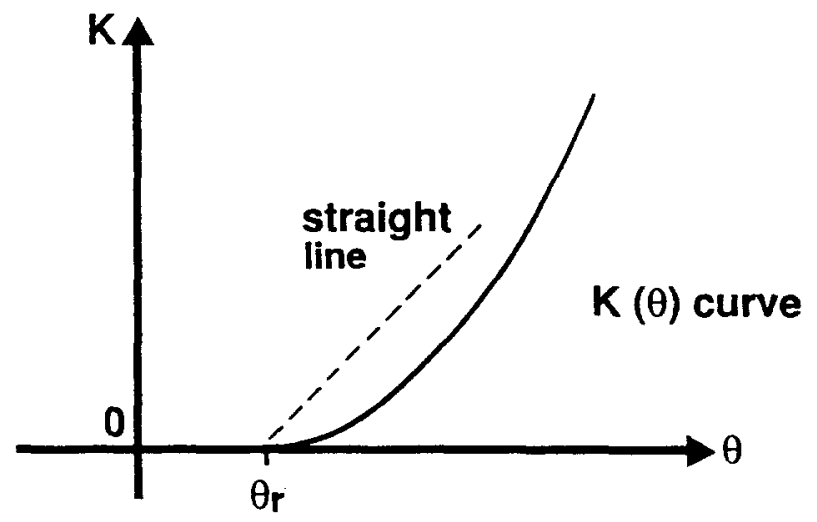

Fig. 6. Condition on $K(\theta)$ for existence of downward wetting-front.

be bounded after an infinitesimal time irrespective of how irregular the initial soil-moisture distribution may be. If the initial soil-moisture velocity is bounded, then the flux will be continuous and the velocity will be bounded from the start.

\section{Properties of Wetting-Fronts}

Continuing the analysis of the occurrence of wetting-fronts, a number of physically relevant properties of the interface $\zeta(t)$ defined by (11) for the solution $u(x, t)$ of the Cauchy problem for Equation (7) have been proven under conditions (12)-(14).

The first of these properties is that there is a minimum speed of propagation. To be specific, it has been proven [15] that for any $t_{1} \geqslant 0$ and $t_{2} \geqslant t_{1}$ there holds

$$
\zeta\left(t_{2}\right) \geqslant \zeta\left(t_{1}\right)+\sigma_{0}\left(t_{2}-t_{1}\right)
$$

where

$$
\sigma_{0}=\limsup _{s \rightarrow 0}-b(s) / s .
$$

In terms of Equation (6), this minimum speed

$$
\sigma_{0}=\limsup _{\theta \rightarrow \theta_{r}}-\lambda \frac{K(\theta)}{\theta-\theta_{\mathrm{r}}}=-\lambda K^{\prime}\left(\theta_{\mathrm{r}}\right)
$$

assuming that the latter exists. Thus, when $\lambda=-1$ or when $\lambda=0$, by the nonnegativity of the hydraulic conductivity function, one has $\sigma_{0} \geqslant 0$. Whereas when $\lambda=1$, the minimum speed $\sigma_{0}<0$ if $K^{\prime}\left(\theta_{\mathrm{r}}\right)>0$, and $\sigma_{0}=0$ if $K^{\prime}\left(\theta_{\mathrm{r}}\right)=0$.

This means automatically that in a vertical profile any wetting-front demarcating the lower boundary of a wetted region cannot move upwards, whilst in a horizontal profile any wetting-front must propagate outwards. Furthermore, any upper wetting-front demarcating a wetted region in a vertical profile cannot move downwards if one can say that $K^{\prime}\left(\theta_{\mathrm{r}}\right)=0$. See Figure 7. Subsequently, since the evidence indicates that $K(\theta)$ relationships have a convex form such as that shown in Figures 


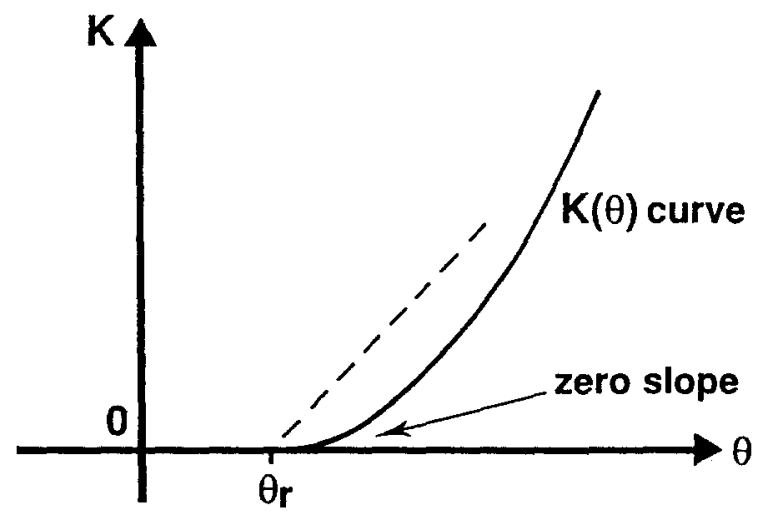

Fig. 7. Condition on $K(\theta)$ for non-contraction of wetting-front.

3, 6 and 7, whatever the direction, wetted regions cannot shrink. The name wetting-front is consequentially not misplaced.

The above answers the question: Is soil-moisture retained? A consequence of the observation that a wetted region can only expand is that at any given place once the water content has increased above its irreducible level it can never again drop back to this level. Consequently, there is always some retention, albeit in real terms perhaps in increasingly smaller concentrations in increasingly narrower pores.

The next question which can be answered by the mathematical theory of (7) is: Does the movement of a wetting-front exhibit jumps? Casting this question in the negatory form for Equation (7) it becomes: Is the interface $\zeta(t)$ continuous? The answer here is: Yes, if the $\sigma_{0}$ defined by (18) is not infinite in magnitude [15]. Whence, under the previously-mentioned criterion of zero slope of the $K(\theta)$ relationship at the irreducible water level $\left(\sigma_{0}=0\right)$ a wetting-front can indeed only move continuously, i.e. without jumps.

(Whatever the value of $\sigma_{0}$, it is known that the interface $\zeta(t)$ is lower semi-continuous and continuous from the right. However, if $\sigma_{0}=-\infty$, the upper semi-continuity of the interface from the left is still an open mathematical question [15].)

A further property to be considered of the interface $\zeta(t)$ relates to the occurrence of a wetting-front as a material surface. Physically, one would expect that the advance of a wetting-front is determined by the speed at which the water particles forming the front travel. The question to be posed then is: Does a wetting-front move at a speed equal to the velocity of the water at the front?

In terms of the solution of the Cauchy problem for Equation (7) this question can be reformulated as: Is

$$
\zeta^{\prime}(t)=\lim _{x \uparrow \zeta(t)}\left[-\left((a(u))_{x}+b(u)\right) / u\right](x, t)
$$

for any $t \geqslant 0$ ? The answer to this question is: Yes, in some limited sense. For details, see $[15,25]$. However, as yet with the exception of the case when $b \equiv 0$ and 
$a$ takes on an idealized form $[2,19]$, here the mathematical theory is far from being complete.

\section{Penetration}

The final question to be addressed in this survey is that of the penetrability of water in a soil. Supposing that one introduces moisture into a restricted region of a dry soil, one may ask: How far will the moisture eventually infiltrate, or, Is moisture penetration boundless?

To answer this last question, an alternative mathematical problem to that previously considered will be examined. This is the Cauchy-Dirichlet problem of solving Equation (7) in the domain

$$
H=\{(x, t): 0<x<\infty, 0<t<\infty\}
$$

subject to the initial condition

$$
u(x, 0)=u_{0}(x) \text { for } 0 \leqslant x<\infty
$$

and the boundary condition

$$
u(0, t)=0 \text { for } 0<t<\infty
$$

Here $u_{0}(x)$ is hypothesized to be a given bounded nonnegative continuous function for $0 \leqslant x<\infty$ which is compatible with the boundary condition (19) via the constraint $u_{0}(0)=0$. This problem admits a unique generalized solution, $u(x, t)$, and defining

$$
\zeta(t)=\sup \{x: 0 \leqslant x<\infty, \quad u(x, t)>0\},
$$

when $\zeta(0)<\infty$ and (13) and (14) hold, it is known that $\zeta(t)<\infty$ for all $t>0$ [15-17]. Since plainly if $u_{0}$ is identically zero, the solution to this problem is $u \equiv 0$, it will be supposed that $\zeta(0)>0$.

With regard to the original question, the above problem may be viewed as the worst possible case. For, in essence, it embodies a semi-infinite profile in which the soil is desiccated, i.e. $\theta=\theta_{\mathrm{r}}$, at the only accessible boundary.

For the new problem the question is: Does

$$
\zeta(t) \rightarrow \infty \text { as } t \rightarrow \infty \text { ? }
$$

The following has been proven [17]:

(i) If

$$
b(s) \leqslant 0 \text { for } 0<s \leqslant s^{*}
$$

for some $s^{*}>0$, then (20) holds. 
(ii) If

$a^{\prime}(s) / s$ is integrable from 0 to $s^{*}$,

$a^{\prime}(s) /\left(\int_{0}^{s} a^{\prime}(r) / r \mathrm{~d} r\right)$ is bounded for $0<s \leqslant s^{*}$

and

$b^{\prime}(s) /\left(\int_{0}^{s} a^{\prime}(r) / r \mathrm{~d} r\right)$ is bounded for $0<s \leqslant s^{*}$ for some $s^{*}>0$, then (20) holds.

(iii) If

$$
b(s)>0 \text { for } 0<s \leqslant s^{*}
$$

and

$$
a^{\prime}(s) / b(s) \text { is integrable from } 0 \text { to } s^{*}
$$

for some $s^{*}>0$, then (20) does not hold.

Otherwise, when the parameter $\sigma_{0}$ defined by (18) is zero, the question is open.

The tools with which these results have been proven are the comparison principle for generalized solutions of (7) mentioned earlier and the integral identity for solutions of the Cauchy-Dirichlet problem

$$
\int_{0}^{\infty} x u(x, t) \mathrm{d} x=\int_{0}^{\infty} x u_{0}(x) \mathrm{d} x-\int_{0}^{t} \int_{0}^{\infty} b(u(x, \tau)) \mathrm{d} x \mathrm{~d} \tau
$$

for any $t>0$. The latter may be construed as a statement of conservation of momentum for the corresponding flow described by the Richard's equation.

Since $b$ in (7) is identified with $\lambda K(\theta)$ in (6) and $K$ is always nonnegative, the condition (21) is automatically fulfilled by Equation (6) when $\lambda=-1$ and when $\lambda=0$. Thus, the result (i) above implies that irrespective of the soil characteristics, for soil-moisture infiltration in the direction of gravitational pull or in a horizontal direction, the penetration of water cannot be limited. In other words, under circumstances in which soil-moisture infiltration can be regarded as a one-dimensional phenomenon, once moisture is introduced into a vertical profile lower-lying regions will without fail become wetted at some later time. Similarly, once water is introduced into a horizontal soil profile, every point will always eventually become wetted.

The second result (ii) above indicates that the penetration of moisture also cannot be limited in an upward direction when the soil-moisture diffusitivity and hydraulic conductivity relations fulfil the implied constraints. The constraints themselves are not at all preposterous. They are, for instance, satisfied by the closed-form $D(\theta)$ and $K(\theta)$ relationships discussed in $[12,13]$. By way of speaking, in soils whose characteristics conform to these constraints, capillary suction is a sufficiently powerful mechanism with regard to upward water penetration to override the influence of downward gravitational pull. 
The final result (iii) above is especially curious with regard to the Richards equation. When $\lambda=1, a^{\prime} / b$ corresponds exactly with

$$
D(\theta) / K(\theta)=\frac{\mathrm{d} h}{\mathrm{~d} \theta}(\theta)
$$

in (6). So, fulfilment of condition (22) means that $h(\theta)$ must tend to some bounded limit as $\theta$ tends to $\theta_{\mathbf{r}}$. The latter though appears to be contradicted by the $h(\theta)$ relationships to be found in the literature $[3,9,12,13,20,24]$ where $h$ disappears from the picture at low water contents. Cf. Figure 2.

Should pressure head $h$ remain bounded as water content $\theta$ approaches its critical level $\theta_{\mathrm{r}}$, then the result (iii) above infers that soil-moisture penetration in an upward direction may be limited under the application of appropriate boundary conditions. However, since the generally exhibited behaviour of the $h(\theta)$ relationship does not conform to this picture, the answer to the question 'Do wetting-fronts expand without bound' is as yet unresolved for the upward moving wetting-fronts in a vertical profile whose characteristics are not covered by (ii).

\section{Discussion and Conclusions}

Nowadays, the use of the Richards equation as a model for the flow of water in an unsaturated soil is commonplace $[4,11-13]$. Dependent on the application involved, it may appear in the form considered here or formulated in terms of pressure head or suction, for a homogeneous or heterogeneous soil, and with or without plant uptake.

The essential difficulty with the mathematical analysis of the Richards equation is the possible occurrence of wetting-fronts where the equation may not be solvable in the classical sense. The actuality of this possibility is determined by the behaviour of the $K(\theta)$ and $h(\theta)$ relationships as the water content approaches its irreducible level. It is of course questionable how relevant the Richards equation is for describing soil-moisture flow when the water content is extremely low. Among the factors neglected in the derivation of Equation (6) is the movement of water in vapour form which becomes influential at low moisture contents. Perhaps more importantly, the validity of the continuum assumption is open to question at this level. A discussion of various aspects of the justifiability of the Richards equation as a model for the flow of water in an unsaturated soil from the view-point of a soil-physicist can be found in [20]. One point which should be highlighted is that of the (un) feasibility of performing experiments to determine $K(\theta)$ and $h(\theta)$ relationships at arbitrary low water contents.

Notwithstanding the above remarks, the mathematical analysis surveyed in this paper has shown that in the form considered here the Richards equation can be solved in a meaningful way. Moreover, it has led to the identification of criteria on the $K(\theta)$ and $h(\theta)$ relationships which characterize the occurrence and the behaviour of wetting-fronts. 
The kind of analysis surveyed in this paper does not only serve to justify the use of such an equation as a model. In practice, experience has repeatedly taught that an understanding of the mathematical properties of an equation is an essential key to the development of efficient computational schemes for the numerical solution of the equation in hand.

\section{References}

1. Alt, H. W. and Luckhaus S., 1983, Quasilinear elliptic-parabolic differential equations, Math. Z. 183, $311-341$.

2. Aronson, D. G., 1986, The porous medium equation, in A. Fasano and M. Primicerio (eds.), Nonlinear Diffusion Problems, Lecture Notes in Mathematics 1224, Springer-Verlag, Berlin, pp. 1-46.

3. Bear, J., 1972, Dynamics of Fluids in Porous Media, American Elsevier, New York.

4. Bear, J., 1979, Hydraulics of Groundwater, McGraw-Hill, New York.

5. Bear, J., Zaslavsky, D., and Irmay, S., 1968, Physical Principles of Water Percolation and Seepage, United Nations Educational Scientific and Cultural Organization, Paris.

6. Bénilan, P. and Touré, H., 1984, Sur l'équation générale $u_{t}=\varphi(u)_{\mathrm{xx}}-\psi(u)_{\mathrm{x}}+v, C$. R. Séances Acad. Sci. Série I Math. 299, 919-922.

7. Cannon, J. R. and Mohamed, F. A., 1989, A multifree boundary problem arising in the theory of liquid flow in a porous medium, Unione Mat. Ital. Boll. B Serie VII 3, 69-93.

8. Chandrasekhar, S., 1943, Stochastic problems in physics and astronomy, Rev. Modern Phys. 15, 1-89.

9. Childs, E. C., 1969, An Intraduction to the Physical Basis of Soil Water Phenomena, Wiley, London.

10. Di Benedetto, E. and Showalter, R. E., 1981, Implicit degenerate evolution equations and applications, SIAM J. Math. Anal. 12, 731-751.

11. Feddes, R. A., Kowalik, P. J., and Zaradny, H., 1978, Simulation of Field Water Use and Crop Yield, Centre for Agricultural Publishing and Documentation, Wageningen, The Netherlands.

12. Genuchten, M. T. van, 1980, A closed-form equation for predicting the hydraulic conductivity of unsaturated soils, Soil Sci. Soc. Amer. J. 44, 892-898.

13. Genuchten, M. T. van and Nielsen, D. R., 1985, On describing and predicting the hydraulic properties of unsaturated soils, Ann. Geophys. 3, 615-624.

14. Gilding, B. H., 1977, A nonlinear degenerate parabolic equation, Ann. Scuola Norm. Sup. Pisa Cl. Sci. 4 4, 393-432.

15. Gilding, B. H., 1988, The occurrence of interfaces in nonlinear diffusion-advection processes, Arch. Rational Mech. Anal. 100, 243-263.

16. Gilding, B. H., 1989, Improved theory for a nonlinear degenerate parabolic equation, Ann. Scuola Norm. Sup. Pisa Cl. Sci. 4 16, 165-224.

17. Gilding, B. H., 1989, Localization of solutions of a nonlinear Fokker-Planck equation with Dirichlet boundary conditions, Nonlinear Anal. Theory Meth. Appl. 13, 1215-1240.

18. Gilding, B. H. and Kersner, R., 1992, The characterization of reaction-convection-diffusion processes by travelling waves, in preparation.

19. Kalashnikov, A. S., 1987, Some problems of the qualitative theory of non-linear degenerate second-order parabolic equations, Russian Math. Surveys 42, 169-222. Translation of Uspekhi Mat. Nauk 42, 135-176.

20. Philip, J. R., 1969, Theory of infiltration, in Ven Te Chow (ed.) Advances in Hydroscience Volume 5, Academic Press, New York, pp. 215-296.

21. Richards, L. A., 1931, Capillary conduction of liquids through porous mediums, Physics 1, 318-333.

22. Sacks, P. E., 1983, Continuity of solutions of a singular parabolic equation, Nonlinear Anal. Theory Meth. Appl. 7, 387-409.

23. Smoller, J., 1983, Shock Waves and Reaction-Diffusion Equations, Springer-Verlag, New York.

24. Swartzendruber, D., 1969, The flow of water in unsaturated soils, in R. J. M. de Wiest (ed.), Flow through Porous Media, Academic Press, New York, pp. 215-292.

25. Wang, S., 1983, The lateral boundary of the support of weak solutions of the filtration equation in one dimension, Acta Math. Sinica (Beijing) 26, 199-219. 